\title{
DOE|ER/10690--12
}

\section{TECHNICAL PROGRESS REPORT OF \\ THE UNIVERSITY OF SOUTH CAROLINA'S \\ HIGH ENERGY PHYSICS GROUP}

'r.

Table of Contents

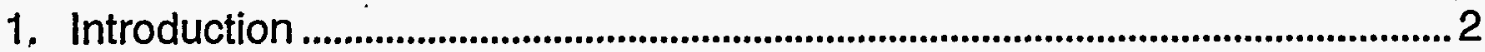

2. The AMY Program ......................................................................................................2

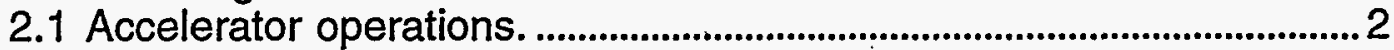

2.2 Trigger upgrade progress........................................................................................ 3

2.3 Personnel and recent activities........................................................................ 5

2.4 The program for 1993........................................................................................ 6

3. Fermilab Experiment E789 ................................................................................. 6

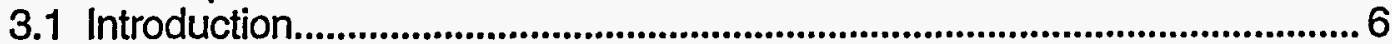

3.2 Performance of the Ring Imaging Cherenkov Counter........................... 6

3.3 Personnel and current activities.....................................................................

3.4 Future of the program ....................................................................................... 9

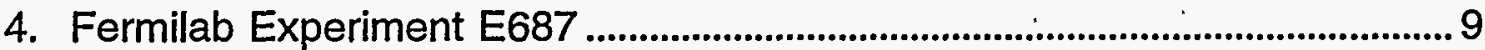

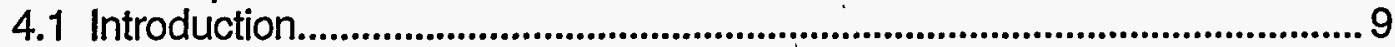

4.2 Personnel and current activities ..............................................................10

4.3 Future of the program ............................................................................... 12

5. Search for the $17 \mathrm{keV}$ neutrino..........................................................................12

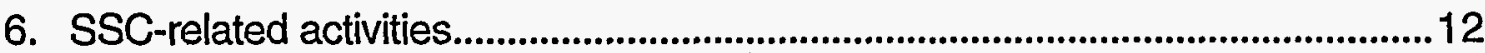

7. Fermilab Proposal P803.......................................................................................13

8. Recent papers co-authored by group members...............................................16

\section{DISCLAIMER}

This report was prepared as an account of work sponsored by an agency of the United States Government. Neither the United States Government nor any agency thereof, nor any of their employees, makes any warranty, express or implied, or assumes any legal liability or responsibility for the accuracy, completeness, or usefulness of any information, apparatus, product, or process disclosed, or represents that its use would not infringe privately owned rights. Reference herein to any specific commercial product, process, or service by trade name, trademark, manufacturer, or otherwise does not necessarily constitute or imply its endorsement, recommendation, or favoring by the United States Government or any agency thereof. The views and opinions of authors expressed herein do not necessarily state or reflect those of the United States Government or any agency thereof. 


\section{DISCLAIMER}

Portions of this document may be illegible in electronic image products. Images are produced from the best available original document. 


\section{Introduction}

The high energy physics group at the University of South Carolina includes five teaching faculty members, one research faculty member, and five graduate students. The faculty are Professors F.T. Avignone, R.L. Childers, and C.W. Darden, Associate Professor C..Rosenfeld, Assistant Professor J.R. Wilson, and Research Assistant Professor A.T.M. Wang. Prof. Avignone works primarily in the area of low energy tests. of fundamental interactions, with a present focus on double beta decay in germanium. The germanium research is not supported by this grant. Professors Childers, and Darden devote most of their research effort to Fermilab experiment E789, which is designed to observe charmless two-body decays of $b$-flavored mesons and baryons. Prof. Wilson works on E789 and also on Fermilab experiment E687 which studies charm physics in the wide-band photon beam. Professors Rosenfeld and Wang participate in the AMY Collaboration, which studies $e^{+} e^{-}$interactions using the TRISTAN collider at KEK. Prof. Rosenfeld and one student collaborate with personnel from KEK and INS, Tokyo, on an experiment to detect a $17 \mathrm{keV}$ neutrino in the $\beta$-decay spectrum of ${ }^{63} \mathrm{Ni}$. Prof. Wang currently holds a visiting appointment at SSC Lab where she is working on projects in which USC is not involved. SSCL pays her salary via a contract with USC. Members of the group also participate in several new initiatives which are described below.

\section{The AMY Program}

\subsection{Accelerator operations.}

Two years ago the TRISTAN interaction regions were equipped with superconducting quadrupoles to improve the luminosity. In the first year following installation, problems associated with the new equipment seriously impeded operations. In consequence, between August 1990 and July 1991 AMY logged just $8 \mathrm{pb}^{-1}$ - only TRISTAN's first year was less productive. The major problems induced by the superconducting quads were eventually resolved, and a welcome improvement in luminosity resulted. By 1 July 1992 AMY was able to log over $700 \mathrm{nb}^{-1}$ on a typical day, and from August 1991 to July 1992 the integrated luminosity was $90 \mathrm{pb}^{-1}$. The year's production was a factor of three greater than the best previous year, and it raises the integrated luminosity since first beam from 70 to $160 \mathrm{pb}^{-1}$. 
None of this performance increment is attributable to a smoothly running accelerator. The filling procedure remains erratic, and bringing it under control is one avenue to further improvement of overall performance. In addition the accelerator group is discussing means for increasing the circulating current and thus achieving still higher luminosity. Some advance on both fronts is likely, and the experimenters look forward to another record-setting year.

\subsection{Trigger upgrade progress}

In 1987 the USC group proposed a multifaceted upgrade of the trigger system. This upgrade became part of a package that included new pole-tip iron for the solenoid, new shower and charged particle instrumentation for the pole tips, and a vertex detector. At the time of the original trigger design the synthesis of radial tracks from track segments appeared exceedingly difficult given the schedule and resource constraints. We guessed correctly that full track finding was also unnecessary. For the upgrade, however, we designed a device to, achieve radial track synthesis and justified it on the grounds that improvements in luminosity might bring higher backgrounds with which the trigger would have to cope. The original menu for the trigger upgrade listed

(1) global track synthesis (radial track correlator),

(2) a system for more accurately counting the segments in a superlayer,

(3) trigger hardware for the muon chambers which would facilitate a redundant dimuon trigger not dependent on the shower counter,

(4) redefinition of a signal for cosmic ray suppression from the inner tracking chamber (ITC), a straw tube chamber inside the central tracker, in order to improve the efficiency for dimuon events,

(5) track trigger systems for the new endcap tracking chambers (FTC) and vertex detector (VTX), and

(6) new logic for shower counter energy triggers that would operate with reduced background.

Three types of modules constitute the basic building blocks for all of these improvements. One is a track segment recognition card, a custom module of which 88 were already in service and 50 additional were required. The second is a high density ECL fan-in module, and the last is a CAMAC memory look-up module designated "STML." Fan-in and memory look-up units suitable for our purposes were not offered commercially. The custom design and fabrication of these modules was therefore an integral part of the project. Plans called for fabrication of $40^{\circ}$ of the ECL fan-ins and 50 
STML's. The trigger upgrade was approved by the collaboration and was funded by DOE in 1989.

Most of the installation work was accomplished during 1991, and additional work during June 1992 brought the upgrade project essentially to completion. All of the electronic modules have been fabricated and debugged. Of the ECL fan-in modules 25 are in service in the experiment. Of the STML modules 49 are in service leaving us with only one spare. The fan-in and memory look-up modules are units with rather general applicability [C. Rosenfeld et al., IEEE Transactions in Nuclear Science 39, 848 (1992)], and we have expectations that when AMY has run its course, we will use these modules in future projects.

The new triggers that have been successful as a result of the upgrade are as follows. 1) One new two-track trigger utilizes signals from the radial track correlator. We established that this trigger operates at a lower rate and higher efficiency than its predecessor, which did not use the correlator. 2) We constructed a back-to-back twotrack trigger also utilizing correlator signals. This trigger runs at a somewhat higher rate than the competitive triggers but does not require a signal from an inner tracking chamber, ITC or VTX, for cosmic ray suppression. 3) We included a correlator requirement in two of our older low multiplicity track triggers to reduce their rates. One of these had been deactivated because of excessive rate and was reactivated in June. 4) We implemented a second track segment recognition system for the ITC, which we can use exclusively for cosmic ray suppression and tune for that purpose. The resulting improvement in two-track trigger efficiency should have been only modest. In an independent development, however, many wires in the upper section of the ITC ceased to function, and the improvement from the new subsystem was actually large and critical to our continued ability to extract physics from dimuon events. 5) We created a system that utilizes the endcap shower counter for triggering on dimuon events produced within its acceptance. This system was not contemplated in the original upgrade plans. 6) We installed track segment recognition for two of three trigger superlayers of the forward tracking chamber (FTC). We use an OR of the two superlayers in coincidence with the endcap shower counter to improve the efficiency for dimuon events within the acceptance of the FTC. 7) We installed track segment recognition for the VTX chamber. In view of the degradation in the ITC, we hoped the VTX subsystem could supplement or substitute for the cosmic ray suppression role of the ITC. The VTX trigger electronics performs to specifications, but the chamber itself has not yet operated reliably enough to test its efficacy for cosmic ray suppression. 
In terms of the menu above we completed items 1,2, 4, and 5, and one of our collaborators at KEK implemented item 6 using a design superior to the one we had been considering. Item 3 remains a loose end. We do already have one dimuon trigger that is independent of the shower counter, so a muon chamber trigger signal is not a pressing need. We could not complete this subsystem because it requires six additional STML's. KEK may underwrite a second batch of STML's, and in that case we will eventually tie up this loose end. We also constructed a subsystem that was supposed to detect back-to-back tracks using only the two superlayers of the FTC. We demonstrated that this subsystem would operate at a sufficiently low rate, but we discovered that the cabling of the FTC was not suitably organized for back-to-back detection. Another patch panel (the trigger system already has four). will have to be fabricated to complete the implementation of the FTC back-to-back subsystem.

\subsection{Personnel and recent activities}

The USC personnel in the AMY experiment, in addition to Rosenfeld, are Prof. A. Wang and graduate students S. Lusin, S. Wilson, and L. Zheng. Lusin was in residence at KEK from 1987 until December 1991. As the only USC representative at the Laboratory full time from 1988 to 1990 , he looked after the USC trigger hardware and kept it current with various changes in the detector. For his thesis he has been analyzing the $e^{+} e^{-} \rightarrow \mu^{+} \mu^{-}$and $e^{+} e^{-} \rightarrow \tau^{+} \tau^{-}$channels. In the TRISTAN energy range these final states exhibit asymmetry in the angular distribution as a consequence of $\gamma Z$ interference. Since returning to USC last December he has tied up the loose ends of his analysis and prepared most of the text and figures for his thesis. In August 1992 he took up a research position at University of Wisconsin, Madison, where he will study SSC trigger problems in parallel with completing the thesis. S. Wilson was in residence at KEK for extended periods until January 1992. He manned AMY shifts and assisted with trigger maintenance. He was pursuing an AMY analysis topic, but he decided in March 1991 to shift his effort to the $17 \mathrm{keV}$ neutrino experiment and is now preparing his thesis on that topic. L. Zheng has been in residence at KEK since March 1991. She contributed heavily to installation and commissioning of the trigger upgrade, and she is now the only trigger maintenance person on site. She is working on the channel $e^{+} e^{-} \rightarrow c \bar{c}$ using inclusive techniques, and her preliminary result for the asymmetry is in accord with the standard model. A. Wang was deeply involved in the design and production of the modules for the trigger upgrade. She is now in residence at SSC Lab and is no longer contributing to AMY. The Japanese Ministry of Education (MONBUSHO) supported a one-year research leave for $\mathrm{C}$. Rosenfeld, 
which terminated in January 1992. His major activity during 1991 was installation of the trigger upgrade. Rosenfeld also traveled to KEK for a week in March 1992 and for one month straddling June and July.

\subsection{The program for 1993.}

We plan to continue the AMY program for the next year in pretty much the same manner as we have for the past eight months. L. Zheng will continue in residence at KEK. She will 'shoulder USC's 'responsibility for trigger maintenance as well as pursuing her analysis project. The provision of spare high voltage distribution boards for the central drift chamber remains an outstanding USC commitment. C. Rosenfeld will work to discharge this obligation and also to develop a second version of the STML module. He will make occasional trips to KEK.

\section{Fermilab Experiment E789}

\subsection{Introduction}

E789 was proposed as'a high luminosity experiment optimized for producing and detecting rare two-body decays of beauty mesons. Shortly after the fixed target running period commenced these goals were reexamined in light of the running conditions actually experienced. The experiment was slightly reconfigured to optimize its sensitivity for $B \rightarrow J / \psi+X$ decays. Some running time was also set aside for a search for rare dileptonic charm decays as well as some charm A-dependence studies. The experiment collected nearly $2 \times 10^{9}$ events on tape. Preliminary analysis suggests a $B$ signal of several hundred events in the $J / \psi+X$ mode, sensitivity to dileptonic decays of charm mesons at the 10-5 level, and several thousand reconstructed $D^{\circ} \rightarrow K \pi$ decays for doing $A$-dependence studies.

\subsection{Performance of the Ring Imaging Cherenkov Counter}

South Carolina assumed responsibility for the analysis of the data from the Cherenkov detector as well as the physical maintenance of the counter itself. The first step in the process was to show that the avalanche chambers were actually detecting showers. These chambers had three views: an $\mathrm{X}$-measuring plane and $\mathrm{U}$ and $\mathrm{V}$ planes rotated by \pm 45 degrees with respect to the $X$ plane. By taking all combinations of one hit in each plane and plotting the residual of the $X$ prediction from the $U$ and $V$ planes we see a nice peak centered at zero (Figure 3.1). We interpret this peak as the detection of showers produced by photons. 
Each photon can be associated with a track, and a measurement of the ring radius can be made. A ring plot such as the one shown in Figure 3.2 can be made for each mirror. The mirror unit vectors are shifted until each ring pattern is centered. After alignment, we found our resolution to be about $1.9 \mathrm{~mm}$, somewhat worse than the E605 value of $1.3 \mathrm{~mm}$. The difference in these two numbers is approximately the difference in chromatic dispersion between pure helium and the mixture we used in E789 (90\% helium and 10\% argon).

A plot of ring radius versus track momentum is shown in Figure 3.3. This curve is fit to the Cherenkov threshold function with one free parameter - the index of refraction. Once the index of refraction has been obtained in this manner, we can measure the efficiency of the $\mathrm{RICH}$ by counting the number of photons within $5 \sigma$ of the expected ring radius for a $\beta=1$ particle. In Figure 3.4 is shown a histogram of the number of photons detected for tracks with momentum $>50 \mathrm{GeV}$. The lowest bin contains a contribution from tracks that are not pions, but the rest of the histogram is nicely fit by a Poisson function with a mean of two photons per track. This is a factor of two less than the four photons per track expected for the radiator gas mixture used in E789. We believe our large pedestal suppression threshold (required by unexpected noise problems in the ADC system) caused most of the discrepancy.

\subsection{Personnel and current activities}

Faculty members J.R. Wilson, R.L. Childers, and C.W. Darden along with graduate student D. Franket are the South Carolina personnel involved with E789. Franket has been at Fermilab for the summer and fall of 1992 and is working on run dependencies of the $\mathrm{RICH}$ alignment - a key factor in integrating the $\mathrm{RICH}$ into the charm or beauty analysis. He is also handling some of the day-to-day bookkeeping involved with the PASS1 analysis currently proceeding on the Fermilab IBM RISC farm: Most of the collaboration's efforts so far have been in processing the $B \rightarrow J / \psi$ data sample, but this fall we intend to undertake the analysis of the $B \rightarrow h^{+} h^{-}$sample at South Carolina. Although the first pass processing of this data is being done on the farm at Fermilab, there will still be about 100 data summary tapes which will need to be analyzed locally. 


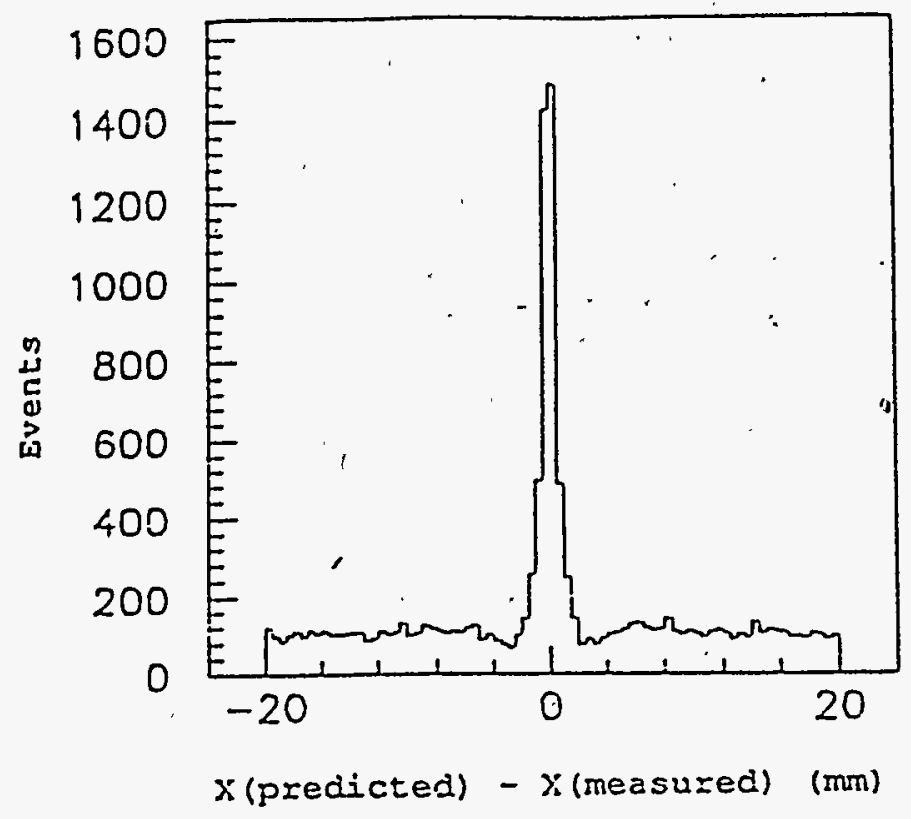

Figure 3.1. Difference between $x$ coordinates predicted by $U-V$ overlaps and hit $x$ wires.

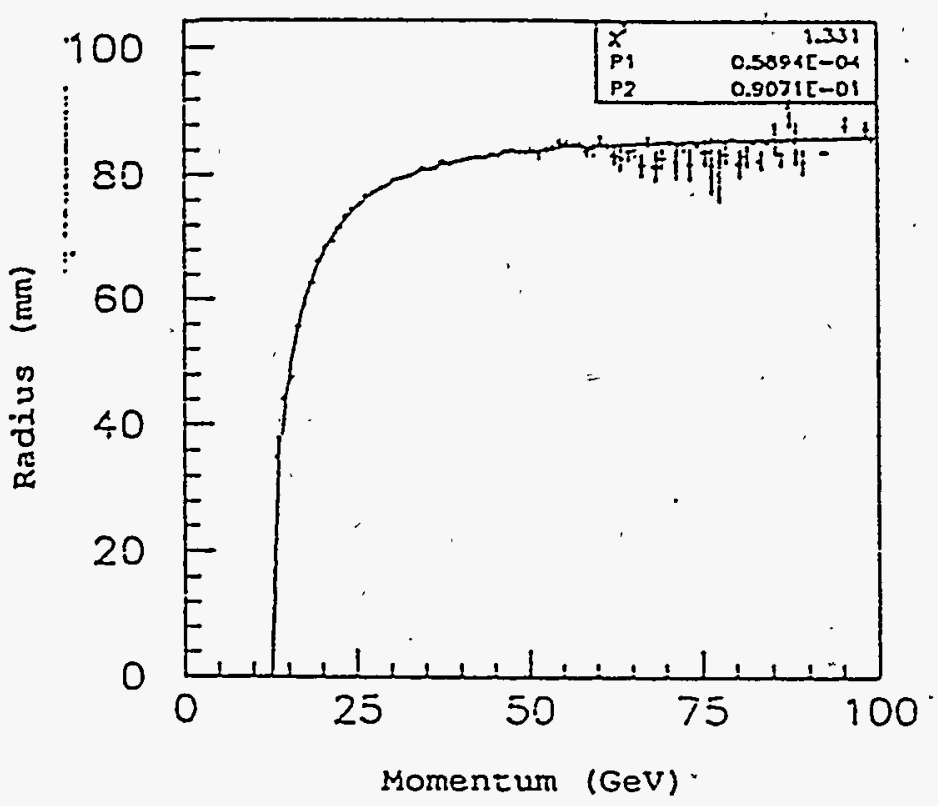

Eigure 3.3. Threshold curve showing radius vezsus momentum. Supezimposed is a fit to. the Chezenkor threshold Eunction.

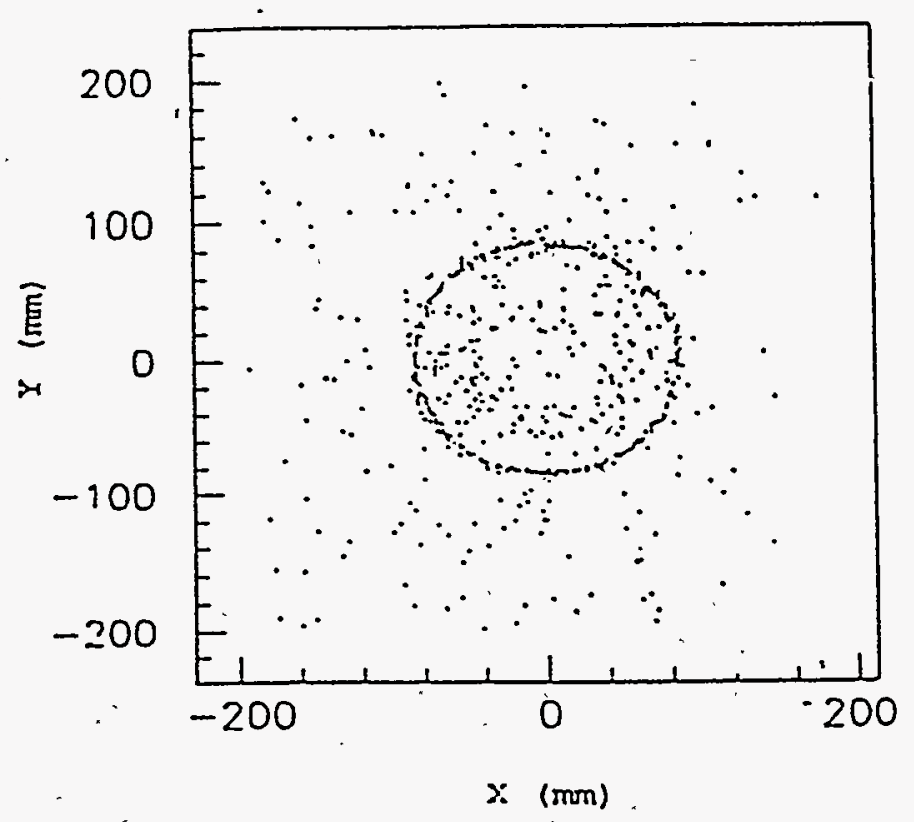

Figure 3.2. Scatter plot of photon position with respect to the ring center integrated over many tracks.

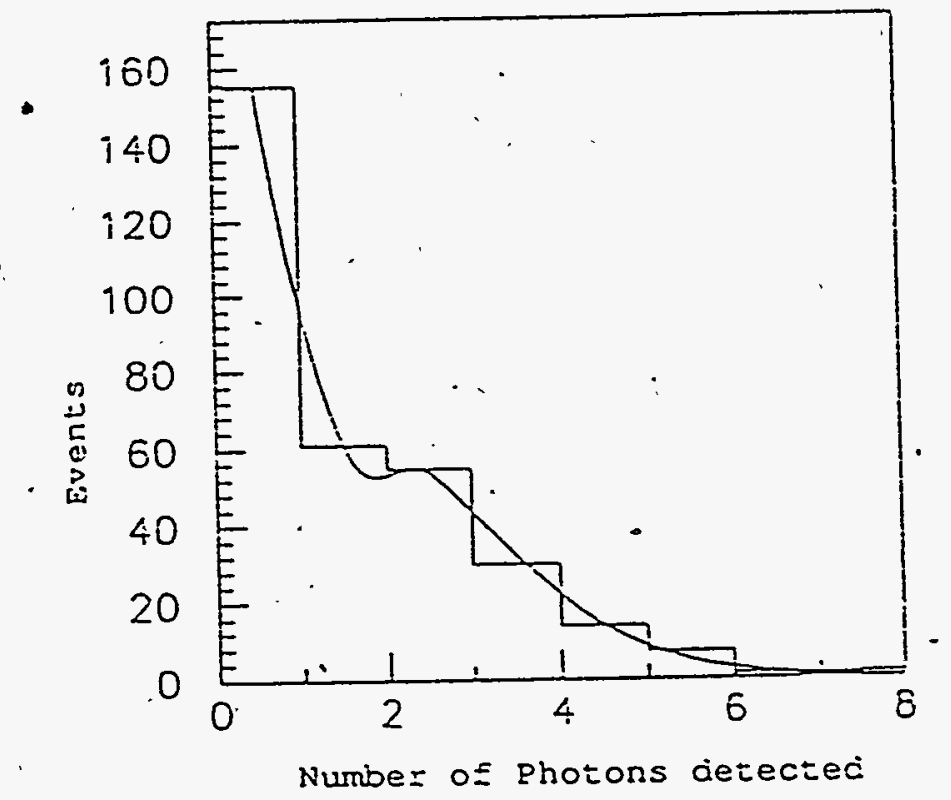

Eigure 3.4. Number of photons detectec pe: track for tracks with momentum greater the $50 \mathrm{GeV}$. A Poisson function rith a mean of 2 photons pez czack is superimposec Ec: bins with $N$ greater than 1 . 


\subsection{Future of the program}

E789 has finished it's scheduled running at Fermilab and the analysis of the data should be finished by next year. Several E789 collaborators are putting together a new proposal based on the E789 high rate spectrometer in order to achieve a two-tothree orders-of-magnitude increase in B production luminosity over the next two fixed target running periods. Childers and Wilson are listed on this proposal.

\section{Fermilab Experiment E687}

\subsection{Introduction}

Experiment E687 is an open spectrometer experiment which ran in the wideband photon laboratory at Fermi National Accelerator Laboratory over two running periods: 1987-88 and 1990-91. The collaboration currently consists of 14 U.S. and foreign institutions including the University of South Carolina.

E687 has reconstructed a sample of about 10,000 charm particle decays from the 1987-88 Fermilab fixed target run. In addition, processing for the 1990-91 run has just been completed and about half of the data has been distributed on data summary tapes to the collaborating institutions. This new sample contains an additional 75,00085,000 charm decays. A histogram containing the "golden" decay modes of the charm mesons $\left(D^{+} \rightarrow K^{-} \pi^{+} \pi^{+}, D^{\circ} \rightarrow K^{-} \pi^{+}, D^{\circ} \rightarrow K^{-} \pi^{+} \pi^{+} \pi^{-}\right)$, which constitute about $80 \%$ of the reconstructable signal, is shown in Figure 4.1. We believe this is currently the largest charm sample in the world.

With a data set of this size it should be possible to measure branching ratios of rare decays modes in the $.01 \%$ range. Many singly Cabibbo suppressed modes have been observed, and studies are underway to do explicit resonance analysis of some of the many-body modes. A comprehensive search is underway for all of the predicted $D^{* \star}$ and $D_{s}^{* *}$ resonances. We are trying to fill in similar gaps in the charmed baryon sector. At the International Conference in Dallas last August (the Rochester Conference) E687 presented confirmation of the $\Omega_{\mathrm{C}}$ baryon containing "ssc" quarks. Precision measurements are being made of the weak decay matrix element in charmed meson semileptonic decays using both muon and electron channels. We should also be able to make similar measurements for the $D_{\mathrm{S}}$ (signal already observed) and $\Lambda_{\mathrm{C}}$ semileptonic decays (search in progress).

- The full dataset of E687 will include more than 500 examples of fully reconstructed photoproduced $D^{-} \bar{D}$ pairs, more by an order of magnitude than used in already 
published studies. Various kinematic features of this sample offer a very clean opportunity for comparison with QCD. For example the acoplanarity of the D's and the incident $\gamma$ is related to the dynamics of gluon radiation. The acoplanarity distribution observed in E687, although in reasonable agreement with the Lund Monte Carlo, is inconsistent with the prediction of leading-order QCD. A calculation of the acoplanarity distribution in next-to-leading order is available for charm hadroproduction, but not yet for photoproduction. We hope the existence of our measurements will encourage some theorist to fill this void.

\subsection{Personnel and current activities}

J.R. Wilson is the USC faculty member associated with E687 and has been a member of the collaboration since 1986. His analysis expertise is in the area of charged particle tracking and neutral kaon analysis. Recently he has studied doubly Cabibbo suppressed decay modes of the $D^{+}$, and using the histogram shown in Figure 4.2, has put a limit on the decay $D^{+} \rightarrow K^{+} \pi^{+} \pi$ relative to the Cabibbo allowed mode $D^{+} \rightarrow K^{-} \pi^{+} \pi^{+}$of 0.007 at the $90 \%$ confidence level. The limit is based on about $50 \%$ of the total data sample and will probably improve when the analysis is complete.

Other studies ongoing at South Carolina are also in the area of rare decays. These include measurements of $D^{+}$and $D_{S}^{+}$decays to four-body states that include two kaons. Other experiments have established that two-body channels account for more than $60 \%$ of the hadronic width of $D$ mesons but do not reveal the corresponding fraction for $D_{\mathrm{s}}$ mesons. We expect our large sample of $D_{\mathrm{s}}$ decays to speak to this issue: 


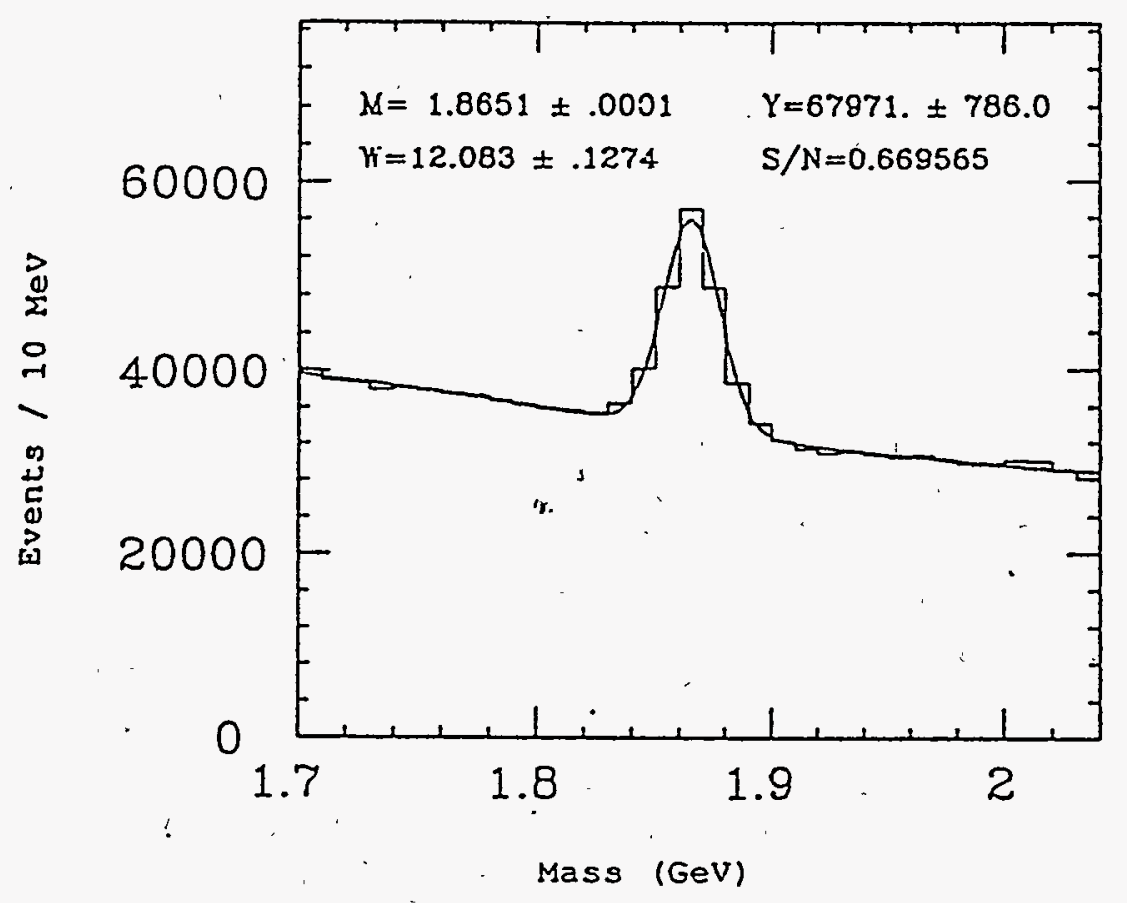

Figure 4.1. Doubly Cabibbo Suppressed channel Dt $-\rightarrow \mathrm{r}+\mathrm{pi}-\mathrm{pit}$. This leads to a $90 \%$ confidence level limit of $1 \%$ for the doubly suppressed channel relative to the allowed channel.

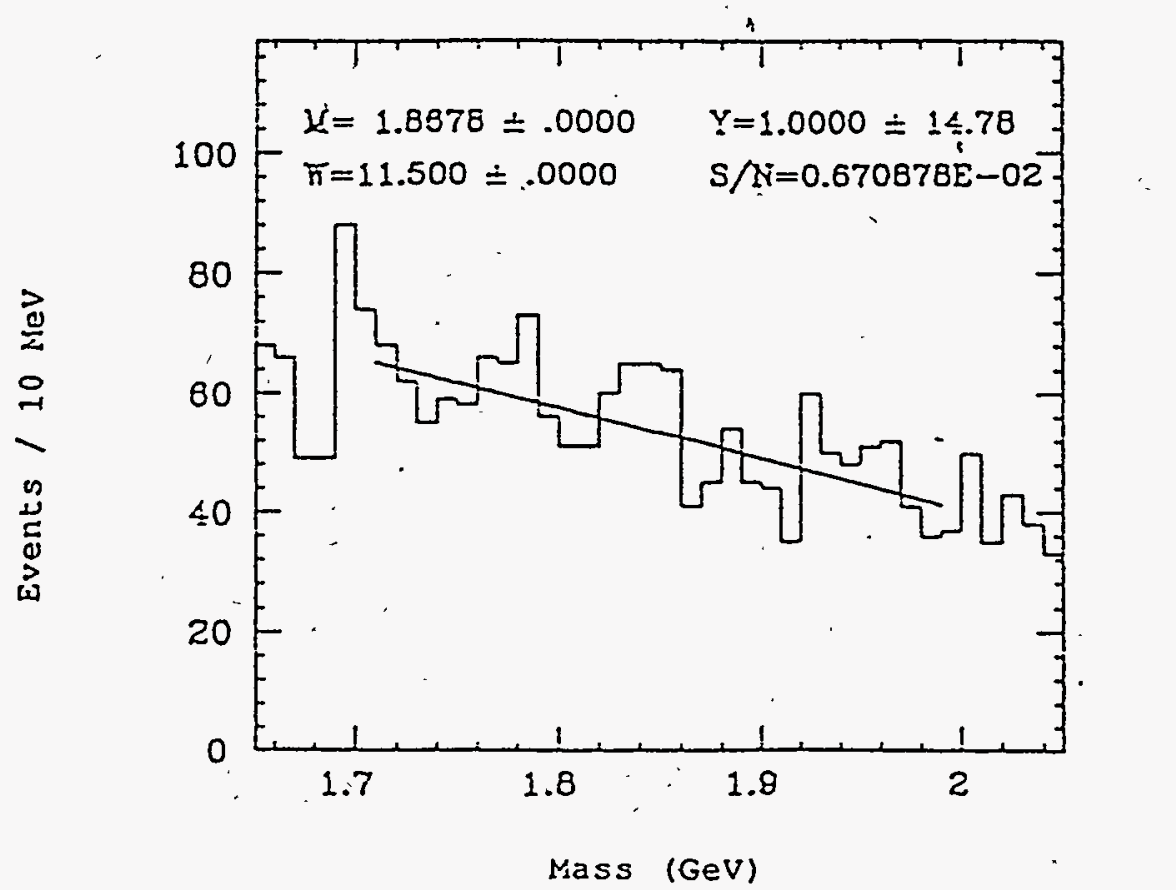

Figure 4.2. Total "Golden" decay mode charm sample for the 1990-91 run of $\overline{6} 667$. 


\subsection{Future of the program}

E687 has concluded its data taking with the 1991 run. The main processing of the data is complete; and it is expected that by the fall of 1992 all of the data reduction will be done as well. By winter the South Carolina HEP group should have a 35-Specmark DEC5000 on which to begin analysis of E687 data summary tapes.

A new proposal is being generated by the E687 collaboration (FNAL P831) to continue the study of the dynamics of photoproduction and rare decays of charm particles. Only very modest changes to the E687 beamline and spectrometer are required to achieve an order-of-magnitude increase in reconstructed charm decays. Wilson is listed on this proposal.

\section{Search for the $17 \mathrm{keV}$ neutrino}

The first claim for the existence of a $17 \mathrm{keV}$ neutrino was based on a measurement of the $\beta$-decay spectrum of tritium published by Simpson in 1985 . Interest in the subject increased dramatically in December 1990 when two papers reporting the spectra of ${ }^{35} \mathrm{~S}$ and ${ }^{14} \mathrm{C}$ confirmed the claim. Prof. T. Ohshima of KEK formed a collaboration to investigate the $17 \mathrm{keV}$ neutrino in ${ }^{63} \mathrm{Ni}$ using an iron-free magnetic spectrometer at the Institute for Nuclear Science, Tokyo (INS). Rosenfeld and graduate student $\mathrm{S}$. Wilson became members of this group.

Data collection was completed in September 1991. An analysis of the data, performed principally by Prof. Ohshima, yielded an upper limit for the branching fraction to a $17 \mathrm{keV}$ neutrino well below the $1 \%$ level associated with Simpson's effect. This result appears in Physics Letters B 287, 45 (1992). S. Wilson is conducting an independent analysis of the data for his Ph.D. thesis.

\section{SSC-related activities}

Our group is participating in detector development work with support from a TNRLC (Texas) infrastructure grant and from a one-year DOE subsystems grant.

The motivations for detector development activities have shifted significantly in the last year. We had been interested in development of a scintillating fiber preradiator and novel devices for fiber light detection. It now appears, however, that neither SDC nor GEM will include a fiber preradiator. The more-or-less definite commitments to imaging fiber technology at SSC are a shower-max detector and charged particle 
tracking in SDC. We are not participating in SDC, but we continue to collaborate with Rockefeller and Yale on the development of fiber light detectors for these applications.

A. Wang was a participant in T-841, a beam test of various calorimeter technologies at Fermilab. The data collection terminated in December 1991. So far one report, M. Hulbert et al., SDC Note SDC-92-294, has emerged from this work. This report is being readied for submission to NIM, and a second paper for NIM is also in preparation.

The last year has seen significant progress in development of pixellated photodetectors. Advanced Photonics Inc. has fabricated for us three successful $3 \times 3$ pixel arrays of avalanche photodiodes. We are now arranging with them to develop $8 \times 8$ arrays. The collaboration also contracted with Hughes Aircraft to develop $8 \times 8$ arrays. Hughes encountered some difficulty and has not yet delivered a working device. They now believe they understand their problem and hope to be back in the competition soon .

Our TNRLC grant also supports a project to investigate the management of high voltage distribution via buried traces in printed circuit boards. We aim to reproduce the long term mode of insulation failure in the popular FR-4 material and then to determine if any of several new PCB materials is more rugged. We designed a circuit board especially suited for this study, and a commercial fabricator has delivered the initial lot. We have populated one board, and we are now preparing instrumentation and software for the test bench.

\section{Fermilab Proposal P803}

Profs. Avignone and Rosenfeld are charter members of Fermilab proposal P803 (spokesman, N. Reay of Ohio State). This experiment will search for the oscillation of $v_{\mu}$ to $v_{\tau}$ with sensitivity better by a factor of 40 than previously achieved. The many searches already conducted for neutrino oscillations attest to the fundamental significance of this phenomenon. Were it to be observed, it would imply that neutrinos have mass and that generational mixing is a feature that the leptons share with the quarks.

Our interest in $v_{\mu} / v_{\tau}$ oscillations is stimulated by questions from cosmology as well as particle physics. If the $\tau$ neutrino oscillates and therefore has mass, then it is a candidate for the cosmological dark matter. For a time the idea that a neutrino could play this role ("hot dark matter") was out of favor with modelers, but it seems now to be back in vogue. A neutrino that is to account for $\Omega=1$ should have a mass in the 
vicinity of 10 to $60 \mathrm{eV}$. Direct measurements of the mass of $v_{e}$ have yielded interesting upper limits, but more importantly, in the Mikheyev-Smirnov-Wolfenstein (MSW) explanation of the solar neutrino problem both the $e$ and $\mu$ neutrino masses are a small fraction of an $\mathrm{eV}$. The solar neutrino problem itself shows no signs of abating. The deficit in Davis's experiment has persisted for many years, and the early reports from the SAGE and GALLEX experiments are both confirmatory. So we must seriously consider MSW, and in that case the remaining neutrino candidate for the dark matter is $v_{\tau}$. At Singapore '90 D. Schramm offered his opinion that the $\tau$ neutrino was the most likely of the identifiable dark matter candidates. If the mass of $v_{\tau}$ is at least $10 \mathrm{eV}$, P-803 achieves its optimum sensitivity in' the mixing angle, reaching down to $\sin ^{2}(2 \alpha)=10^{-4}$.

The new 120-GeV Main Injector for the Tevatron will be the source of the beam for this experiment. The extracted protons will interact in a thick target, and a double-horn system will focus the resulting pions and kaons into a decay pipe extending 300 to $500 \mathrm{~m}$. The neutrinos exiting the pipe will pass through $100 \mathrm{~m}$ of earth and iron shielding on their way to the detector. This beam line is similar in concept to old designs, but the extraordinary intensity and repetition rate of the accelerator place heavy demands on the target, the horns, and the radiation containment. The high intensity, however, is essential to the sensitivity of P.803 and is the reason that the experiment is totally dependent on the new injector. This beam line is likely to be shared with an associated "long baseline" experiment that will further add to its complexity. The beam line cost is expected to be at least $\$ 20$ million, more than twice the cost of the experiment's detector.

In P803 we will search for the explicit appearance of a $\tau$. Since $c \tau_{\tau}=0.1 \mathrm{~mm}$, the neutrino target must have roughly 10 -micron spatial resolution. Therefore the neutrino target is nuclear emulsion, which is the only proven detector that provides sufficient resolution in a large mass at acceptable cost. For track-following in the emulsion to proceed at a reasonable pace, the process must receive guidance from tracking chambers. We believe this guidance must include a position measurement of a few tracks in each event with a precision of order $200 \mu \mathrm{m}$ where the tracks exit the emulsion stack. Extrapolating trajectories from downstream drift chambers does not yield such high precision. The $\mathrm{P} 803$ proposal envisions acquiring this measurement from scintillating fibers immediately adjacent to the emulsion. Fiber technology, however, has some severe problems. A fiber readout is expected to cost between $\$ 1.5$ million and $\$ 3.0$ million, and the combination of resolution and light output from 
today's fibers is at best marginal for our application. The collaboration is interested in alternative technologies, and South Carolina would like to pursue one such possibility. The likelihood of success is not great, but the prospect of a large payoff justifies a modest effort. Our budget this year includes $\$ 8 \mathrm{~K}$ of equipment funds for this development work of which the University will contribute half.

The Fermilab management has put P803 in "deferred" status even though the science of this proposal has received generally high marks from the Fermilab program committee and other reviewers. Uncertainty of funding for the Main Injector itself has for the last 18 months cast a dark shadow over dependent experiments. Now that the Congress has voted construction funds for the Main Injector in two successive years, its future is probably secure. We now expect laboratory'management to start P803 through the budgetary process. We are confident that if we are patient, the experiment will be approved in due course. 


\section{Recent papers co-authored by group members}

Observation of the Decays $D_{s}^{-} \rightarrow \phi e^{-\nabla}$ and $D^{-} \rightarrow K^{* 0} e^{--\nabla}$. ARGUS Collaboration ( $H$. Albrecht et al.).

Phys. Lett. B255, 634 (1991).

A Spin Parity Analysis of $\gamma \rightarrow \rho^{+} \rho^{-}$. ARGUS Collaboration ( $H$. Albrecht et al.). Phys. Lett. B267, 535, (1991).

Observations of $\Lambda_{c}^{+}$Semileptonic Decay. ARGUS Collaboration ( $\mathrm{H}$. Albreçht et al.). Phys. Lett. B269, 234, (1991).

A Measurement of Asymmetry in the Decay $\Lambda_{c}^{+} \rightarrow \Lambda \pi^{+}$. ARGUS Collaboration $(H$. Albrecht et al.). Phys. Lett. B274, 239 (1992).

First Evidence of $\chi_{c}$ Production in B-Meson Decays. ARGUS Collaboration ( $H$. Albrecht et al.).

Phys. Lett. B277, 209, (1992).

A Measurement of the Inclusive Semileptonic Decay Fraction of Charmed Hadrons. ARGUS Collaboration $(H$. Albrecht et al.). Phys. Lett. B278, 202 (1992).

Inclusive Production of $D^{\circ}, D^{+}$, and $D^{*}+(2010)$ Mesons in $B$ Decays and Nonresonant $e^{+} e^{-}$ Annihilation at $10.6 \mathrm{Gev}$.

ARGUS Collaboration (H. Albrecht et al.).

Z. Phys. C52, 353, (1991).

The Measurement of $D_{s}^{+}$and $D^{+}$Meson Decays Into $\bar{K}^{*+} \bar{K}^{* 0}$. ARGUS Collaboration ( $H$. Albrecht et al.). Z. Phys. C5 $\underline{53}, 361,(1992)$.

Measurement of Exclusive One-Prong and Inclusive Three-Prong Branching Ratios of the Tau Lepton.

ARGUS Collaboratión ( $\mathrm{H}$. Albrecht et al.).

Z. Phys. C53, 367, (1992).

Production of $D_{s}^{+}$Mesons in $B$ Decays and Determination of $F\left(D_{s}\right)$. ARGUS Collaboration ( $\mathrm{H}$. Albrecht et al.).

Z. Phys. C54,1 (1992).

Measurement of $R$ and Determination of the Charged Particle Multiplicity in $e^{+} e^{-A n n i h i l a t i o n ~ a t ~} \sqrt{S}$ Around $10 \mathrm{Gev}$. ARGUS Collaboration $(\mathrm{H}$. Albrecht et al.).

Z. Phys. C54, 13, (1992).

A New Determination of the $B^{\circ}-\bar{B}^{\circ}$ Oscillation Strength.

ARGUS Collaboration ( $H$. Albrecht et al.).

DESY-92-050, to be published.

Evidence for Hard Scattering of Hadronic Constituents of Photons in Photon-Photon Collisions at TRISTAN.

R. Tanaka et al. (the AMY Collaboration).

Physics Letters B277, 215 (1992).

CAMAC Staggered Memory Look-up Module and ECL Fan-in for Fast Trigger Applications. C. Rosenfeld, A.T.M. Wang, S.R. Wilson, L.Y. Zheng, and K.W. Broome. IEEE Transactions on Nuclear Science 39, 848 (1992). 
A Measurement of the $D^{\circ}$ and $D^{+}$Lifetimes.

P.L. Frabetti et al. (the E687 Collaboration).

Physics Letters B263, 584 (1991).

Description and Performance of the Fermilab E687 Spectrometer.

P.L. Frabetti et al. (the E687 Collaboration).

Nuclear Instruments and Methods in Physics Research A $\underline{320}, 519$ (1992).

Measurement of the Decays $D^{\circ} \rightarrow \pi^{+} \pi^{+} \pi^{-} \pi^{-}, D^{\circ} \rightarrow K^{-} K^{+}$, and $D_{s}^{+} \rightarrow \phi \pi^{+} \pi^{+} \pi^{-}$.

P.L. Frabetti et al. (the E687 Collaboration).

Physics Letters, B281, 167 (1992).

Study of $D^{\circ} \rightarrow K_{\mathrm{s}}^{\circ} \pi^{+} \pi^{-}$and $D^{\circ} \rightarrow K_{\mathrm{s}}^{\circ} K^{+} K^{-}$in High Energy Photoproduction.

P.L. Frabetti et al. (the E687 Collaboration).

Physics Letters B286, 195 (1992).

A Measurement of Elastic $J / \psi$ Photoproduction Cross Section at Fermilab E687.

P.L. Frabetti et al. (the E687 Collaboration).

Submitted to Physics Letters B.

High Sensitivity search for a $17 \mathrm{keV}$ Neutrino. Negative Indication with an Upper Limit of $0.095 \%$.

H. Kawakami, S. Kato, T. Ohshima, C. Rosenfeld, H. Sakamoto, T. Sato, S. Shibata, J. Shirai, Y. Sugaya, T. Suzuki, K. Takahashi, T. Tsukamoto, K. Ueno, K. Ukai, S. Wilson, and

Y. Yonezawa.

Physics Letters B287, 45 (1992): 